\title{
Effect of electric potential on the morphology and chemical composition of hollow fiber membrane surface in alkaline medium
}

\begin{abstract}
The impact of electric potential on the dimensional characteristics and membrane surface morphology was investigated in this work. Our study samples comprise hollow fiber (HF) Polysulfone (PS) membrane. Conductive additives have been incorporated within the membrane matrix at the desired predefined concentration. The DC operated electrochemical apparatus consists of graphite anode and stainless steel cathode. The voltage and current density ranges are 2 to 10 volts and 0.01 to $1.4 \mathrm{~mA} / \mathrm{mm}^{2}$ respectively. These investigations have been conducted in alkaline medium at $\mathrm{pH}=8.5$. The results of this work revealed essential changes of hollow fiber (HF) dimensions, including external (DO), internal (DI) and membrane thickness (t). The maximum decrease of DO, DI, and $\mathrm{t}$ were $13 \%, 15 \%$, and $11 \%$ respectively at 5 volts. EDX analysis showed the maximum sodium ions of about $0.84 \%$ on the membrane surface at 2.5 volts after 1 -hour treatment.
\end{abstract}

Keywords: hollow fiber membrane, electrochemical treatment, modification, alkaline medium
Volume 4 Issue 3 - 2019

\author{
Nourhan A Shawky, ${ }^{\prime}$ Aly Abdallah SM, ${ }^{2}$ \\ Mohamed H Sorour,' Ahmed M Awad \\ Abouelata,' Mona A Abdel-Fatah' \\ 'Chemical Engineering and Pilot Plant Dept, Engineering \\ Research Division, National Research Centre, Egypt \\ ${ }^{2}$ Chemical and Refining Engineering Dept, Faculty of Petroleum \\ and Mining Engineering, Suez University, Egypt
}

\author{
Correspondence: Mona A Abdel-Fatah,Assistant Professor, \\ Chemical Engineering and Pilot Plant Dept., Engineering \\ Research Division, National Research Centre, Dokki, Cairo, \\ Egypt, Tel +2 02 33371499/233371728, \\ Email monamamin7@yahoo.com
}

Received: June 17, 2019 | Published: June 28, 2019

\section{Introduction}

Membrane separation is a fundamental unit operation for water treatment and desalination. ${ }^{1}$ Currently, Egypt suffers from water shortage problems due to the increase in water demand and the limited available surface or groundwater supplies. The suggested solution to overcome the current situation is a rationalization of water consumption, adoption of water treatment and reuse programs in addition to water desalination. Water treatment and reuse projects may include numerous membrane separation processes such as microfiltration (MF), ultrafiltration (UF), and nanofiltration (NF). On the other hand, brackish and seawater desalination depends principally on electro-dialysis, nanofiltration and reverse osmosis. With such increasing dependence on membrane separation, it is important to improve the characteristics of separation membrane and enhance membrane reliability. ${ }^{2,3}$ In particular, membrane flux, solute rejection, and fouling come as the priority of membrane development, manufacture, and operation. Polysulfone (PS) material has been used in membrane fabrication due to its excellent physical and chemical properties such as thermal stability, good mechanical strength, and good chemical resistance to several materials in different $\mathrm{pH}$ range. PS membranes applications include water desalination, hemodialysis, wastewater treatment, water desalination, food and drinking processes, membrane distillation and gas separation. ${ }^{4-6}$

Although of these advantages, there are two major drawbacks in the use of PS membrane: fouling and hydrophobic properties. Many studies have been done to overcome these problems by finding new techniques for PS membrane surface modification. ${ }^{7.8}$ Some authors ${ }^{9-14}$ used a combination of electrochemical cell and membrane system for the enhancement of the filtration process during treatment of different wastewater sources and mitigate membrane fouling. Removal efficiencies of COD, orthophosphate, ammonia, and nitrogen compounds using the current density of $12.5 \mathrm{~A} / \mathrm{m}^{2}$ reached to $95 \%$,
$85 \%$, and $80 \%$ respectively using Poly-vinylidene-di-fluoride (PVDF) hollow fiber membrane with stainless steel electrodes. ${ }^{9}$ The removal efficiency of COD was more than $95 \%$ at different voltages tested $0.5,0.7,0.9$ Volt when using Nickel as a cathode and graphite fiber brush as anode. ${ }^{10}$ Membrane filtration and fouling reduction were enhanced by about $44 \%$ at a current density of $0.5 \mathrm{~mA} / \mathrm{cm}^{2}$ applied on HF membrane filtration. The adopted electrodes were aluminum anode and stainless steel cathode. ${ }^{11}$ With the aid of electric current from electrodes composed of the aluminum anode and stainless steel cathode, membrane fouling reduced three times than without using electric current in the treatment of synthetic wastewater. ${ }^{12}$ Applying a voltage of $10 \mathrm{mV}$ on PVDF hollow fiber membrane on filtration of cheese wastewater and synthetic solution showed $90 \%$ removal of COD and $69 \%$ of total inorganic nitrogen compounds with aid of carbon cloth electrodes. ${ }^{13}$ Increasing of flux was observed when electric field strength from 15 to $20 \mathrm{~V} / \mathrm{cm}$ was employed in a reactor consisting of polypropylene hollow fiber membrane and electrodes of stainless steel material in the treatment of urban domestic sewage. ${ }^{14}$ Some studies investigated the effect of electric current as a posttreatment on membrane surface materials. The effect of electric field on (PVDF) microfiltration membranes has been studied and succeeded in imparting piezoelectric properties to the membrane due to the polar effect of the electric field. Filtration experiments of polyethylene glycol (PEG) showed a decrease of fouling and enhancement of membrane flux after polar treatment. ${ }^{15,16}$ Changing membrane microstructure and membrane topography have been confirmed by using electric field treatment on different selected membrane surfaces including; MF, UF, NF, and RO membranes. The results of the treated membranes showed a decrease in surface roughness. Membrane performance was also affected where the filtration experiments showed a flux decline and high rejection. ${ }^{17,18}$ The purpose of the present study is to study the electric field effects on HF membrane surface morphology and chemical compositions. 


\section{Materials and method}

\section{Materials}

Two different dimensions of HF polysulfone (PS) membranes were supplied by National Research Centre (HF project). Membranes (A) and (B) have the same composition. Membrane (A) dimensions, including outside diameter, inside diameter and wall thickness, are $356,172, \& 92 \mu \mathrm{m}$ respectively, and membrane (B) dimensions are 409,223 , and $93 \mu \mathrm{m}$. Sodium acetate (anhydrous, 99\% purity) and acetic acid glacial $(99.7 \%$ purity) were purchased from (Alpha chemika, India).

\section{Electrochemical cell design}

Two electrodes were used with the following dimensions: the diameter and the height of graphite anode were 2.1 and $17 \mathrm{~cm}$ respectively. The corresponding dimensions of stainless steel cathode cylinder diameter were $10.3 \mathrm{~cm}$ and height $13.5 \mathrm{~cm}$. Two-liter beaker was used as the electrochemical cell. The electrolyte volume solution was 1.5 liter in all experiments. A laboratory DC power supply ( $\mathrm{Gw}$ GPR-3030, USA) with range (0-32 voltage) and (0-3.2 Ampere) was used.

\section{Electrochemical treatment}

HF membrane bundles had been fitted vertically in the cell between graphite and stainless steel electrodes, the distance between electrodes was $4.1 \mathrm{~cm}$. The cell was submerged in $2000 \mathrm{ml}$ cylindrical container containing $1500 \mathrm{ml}$ of sodium acetate $(0.1 \mathrm{M})$ as the electrolyte solution. The HF membranes after that were exposed to different electric potential from 2 to 10 volts and from 0.01 to 1.7 amperes, and current density from 0.01 to $1.4 \mathrm{~mA} / \mathrm{mm}^{2}$ in alkaline medium $(\mathrm{pH}=8.5)$ for a time period from 15 minutes to 2 hours. Figure 1 shows the electrochemical cell set-up design. After the treatment, the membranes were rinsed with (RO) water and dried before characterization experiments.

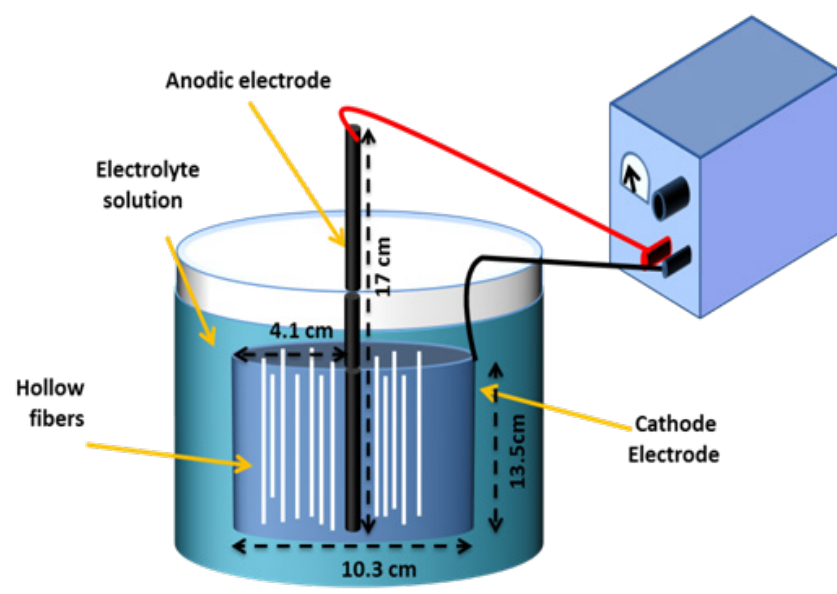

Figure I Experimental set-up.

\section{Characterization and measurement}

\section{Scanning electron microscopy (SEM)}

HF membranes structure details were investigated using (JEOL JCM-6000apparatus). The inner, outer diameters and thickness of membranes were examined by cutting HF membranes to cross- sectional samples using a sharp blade and exposed to sputter device for 0.5 minutes to coat the samples with golden layer and fixed with a paper clip and put inside the stage holder.

\section{Energy-dispersive $\mathrm{X}$-ray spectroscopy (EDX)}

Elemental analysis of the membrane surface was investigated using the EDX device attached to (SEM, JEOL JCM-6000 apparatus). Fibers were cut and put under X-ray spectrum using a probe with high current 15 volt and high vacuum mode.

\section{Results and discussion}

\section{Electric double layer and electrolysis reactions}

Membrane surface when contacted with an aqueous solution, begins to acquire an electric charge. After that electrostatic interaction formed due to the interaction between membrane surface charge and surrounding ions in the solution. ${ }^{19}$ Attraction or repulsion forces occurred depends on the membrane surface charge and charge of ions carried under the effect of the electric field. The distribution of ions in the solution and its interaction with the membrane surface are described by the electric double layer. Dissociation of membrane functional groups depends on the $\mathrm{pH}$ and concentration of the electrolytic solution. According to previously described, PS membrane in this work when coming into contact with sodium acetate ions in the existence of the electric field, membrane surface dissociates to form a negative charge due to the occurrence of special additives. The sodium acetate is decomposed to acetate anions and sodium cations causing the formation of electrostatic forces and adsorbing of sodium ions on the membrane surface. Reactions that formed at the anode and the cathode are:

$$
\begin{gathered}
\mathrm{CH}_{3} \mathrm{COONa} \rightarrow \mathrm{CH}_{3} \mathrm{COO}^{-}+\mathrm{Na}^{+} \\
\mathrm{H}_{2} \mathrm{O} \rightarrow \mathrm{H}^{+}+\mathrm{OH}^{-}
\end{gathered}
$$

Anode (oxidation reaction):

$$
\begin{gathered}
2 \mathrm{CH}_{3} \mathrm{COO}^{-} \rightarrow 2 \mathrm{CH}_{3} \mathrm{COO}^{0} \rightarrow \mathrm{CH}_{3}-\mathrm{CH}_{3}+\mathrm{CO}_{2} \\
\text { (Kolb's reaction) }
\end{gathered}
$$

$$
4 \mathrm{OH}^{-} \rightarrow \mathrm{O}_{2}+2 \mathrm{H}_{2} \mathrm{O}+4 e^{-}
$$

Cathode (reduction reaction):

$$
\begin{gathered}
\mathrm{Na}^{+}+\mathrm{R}-\mathrm{OH}(\text { on membrane surface }) \rightarrow \mathrm{R}-\mathrm{ONa}+\mathrm{H}^{+} \\
2 \mathrm{H}^{+}+2 e^{-} \rightarrow \mathrm{H}_{2}
\end{gathered}
$$

Oxidation reactions are formed at the anode, one for acetic ions where it is oxidized to form ethane gas and carbon dioxide according to Kolb's electrolysis reaction ${ }^{20}$ while the other reaction is the oxidation of hydroxyl ions to form oxygen gases. Reduction reactions are formed at the cathode reaction where the sodium ions are adsorbed on the membrane surface and forming hydrogen ions which are reduced to form hydrogen gas. The reasons for selection graphite electrode as anode due to relatively cheap, good electrical and thermal conductivity and less corrosive in low voltage and current, ${ }^{21}$ while Stainless steel use as cathode due to high corrosion resistance, high conductivity, and mechanical strength, hence combining Carbon and metal materials can help in giving the best results. ${ }^{22}$ 


\section{Effect of electric current on elemental composition}

Chemical composition of untreated HF membrane contained carbon, nitrogen, oxygen, and sulfur. When compared with electrochemically treated HF membranes, new ions of sodium have been observed in all conditions of voltage treatment and a small change in other elementals mass percent. Table 1 shows the mass percent composition of HF membrane before and after electrochemical treatment at $\mathrm{pH}=8.5$.

Table I Mass percentage composition of HF membrane surface in alkaline medium.

\begin{tabular}{llllll}
\hline Mass percent \% & & & & & \\
\hline Volt treatment & $\mathbf{C}$ & $\mathbf{N}$ & $\mathbf{O}$ & $\mathbf{N a}$ & $\mathbf{S}$ \\
Untreated & $47.9 \mathrm{I}$ & 17.23 & 26.6 & 0 & 8.25 \\
2 & 45.49 & 20.4 & 25.46 & 0.22 & 8.43 \\
2.5 & 46.39 & 18.55 & 26.66 & 0.84 & 7.56 \\
5 & 47.79 & 17.4 & 26.23 & 0.38 & 8.18 \\
7.5 & 48.4 & 17.05 & 26.53 & 0.22 & 7.78 \\
10 & 45.75 & 19.26 & 26.65 & 0.19 & 8.17 \\
\hline
\end{tabular}

In an alkaline medium with the increasing voltage applied, the concentration of functional groups of membrane surface increased and negative surface charge increased leading to an electrostatic repulsion between negative ions in the submerging solution and negative charges on membrane surface which has been described previously with electric double layer phenomena. From EDX analysis data, we could conclude that when electric potential is applied on the membrane surface in alkaline medium, hydrogen liberation occurs on the membrane surface and exchange hydrogen ions with the positive charge ion (sodium ions) adsorbed on the membrane surface and slight increase of potential led to the formation of more sodium ions. When potential exceeds 2.5 volts the electrolysis impacts the membrane surface and some sodium ions were stripped away. Although 2.5volt-treatment gives a higher indication of sodium ions in the alkaline medium for one-hour treatment, we studied the time variation at 5 volts to know if it would give the same mass percent of 2.5 -volt treatment at less time or not. Table 2 shows the variation of time at 5 -volt treatment and the higher content was 0.62 after at $30 \mathrm{~min}$ which was less than $0.84 \%$ mass at 2.5 -volt treatment $t$ at 1 -hour.

Table 2 Variation of time at 5-volt treatment in alkaline medium.

\begin{tabular}{llllll}
\hline Volt treatment & \multicolumn{5}{l}{ Mass percent \% } \\
\hline & $\mathbf{C}$ & $\mathbf{N}$ & $\mathbf{O}$ & $\mathbf{N a}$ & $\mathbf{S}$ \\
\hline Untreated & 47.91 & 17.23 & 26.6 & 0 & 8.25 \\
$15 \mathrm{~min}$ & 43.2 & 19.37 & 27.14 & 0.36 & 7.44 \\
$30 \mathrm{~min}$ & 44.64 & 19.73 & 27.46 & 0.62 & 7.55 \\
$45 \mathrm{~min}$ & 45.71 & 19.78 & 25.92 & 0.36 & 8.23 \\
$60 \mathrm{~min}$ & 47.79 & 17.4 & 26.23 & 0.38 & 8.18
\end{tabular}

Effect of electric current on the morphology of HF membranes surface

SEM images showed that during EC and with the increasing voltage applied over 2.5 volts, more cracks are observed on the membrane surface. Cracks formation probably due to the high mobility and movement of ions in the electrolyte solution with increasing electric current and applied voltage. Deformation of the surface and cracks were much bigger in 10-volt treatment. Figure 2 shows cracks formed on the HF membrane surface for different voltages applied in the alkaline medium.

Effect of time on membrane surface has been studied where; membrane treated at 5 volts in alkaline medium at $15,30,45$, 60 minutes showed that cracks and deformation began to be formed after 15 minutes on the membrane surface. Figure 3 shows the time effect on the membrane outer surface in 5-volt treatment.

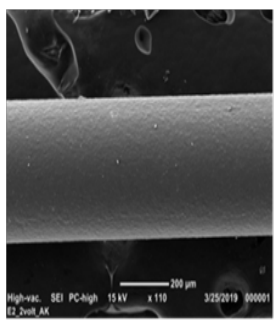

(a)

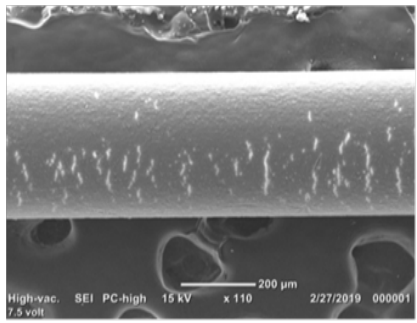

(d)

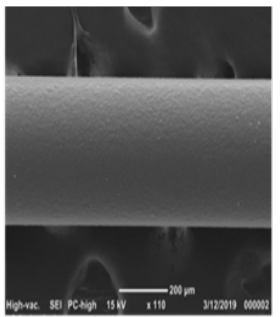

(b)

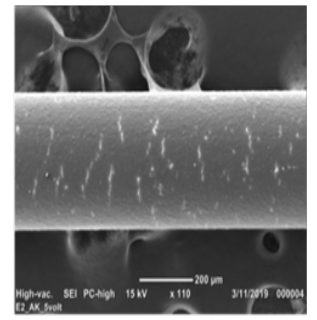

(c)
Figure 2 Electrically treated membrane surface at different voltage in alkaline medium $\mathrm{pH}=8.5$.

(A) 2-volt treatment (B) 2.5-volt treatment (C) 5-volt treatments (D) 7.5-volt treatment (E) I0-volt treatment

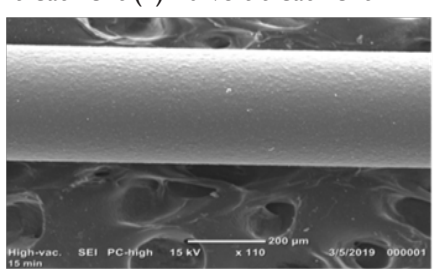

(a)

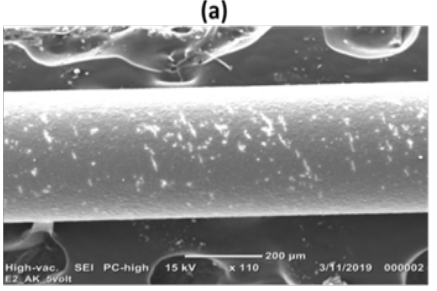

(c)

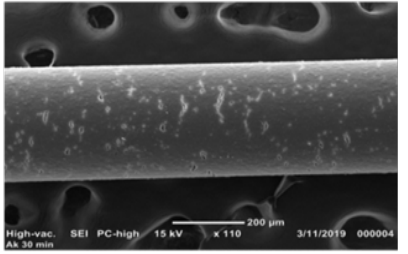

(b)

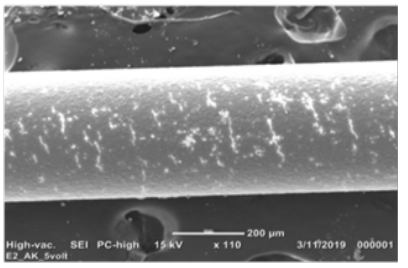

(d)
Figure 3 Time effect on the membrane outer surface in the 5-volt treatment in alkaline medium.

SEM data showed that electric field forces have the ability to change membrane dimensions (DO, DI, and thickness). With the increase of electric current, membrane dimensions decreased as shown in Figure 4. 

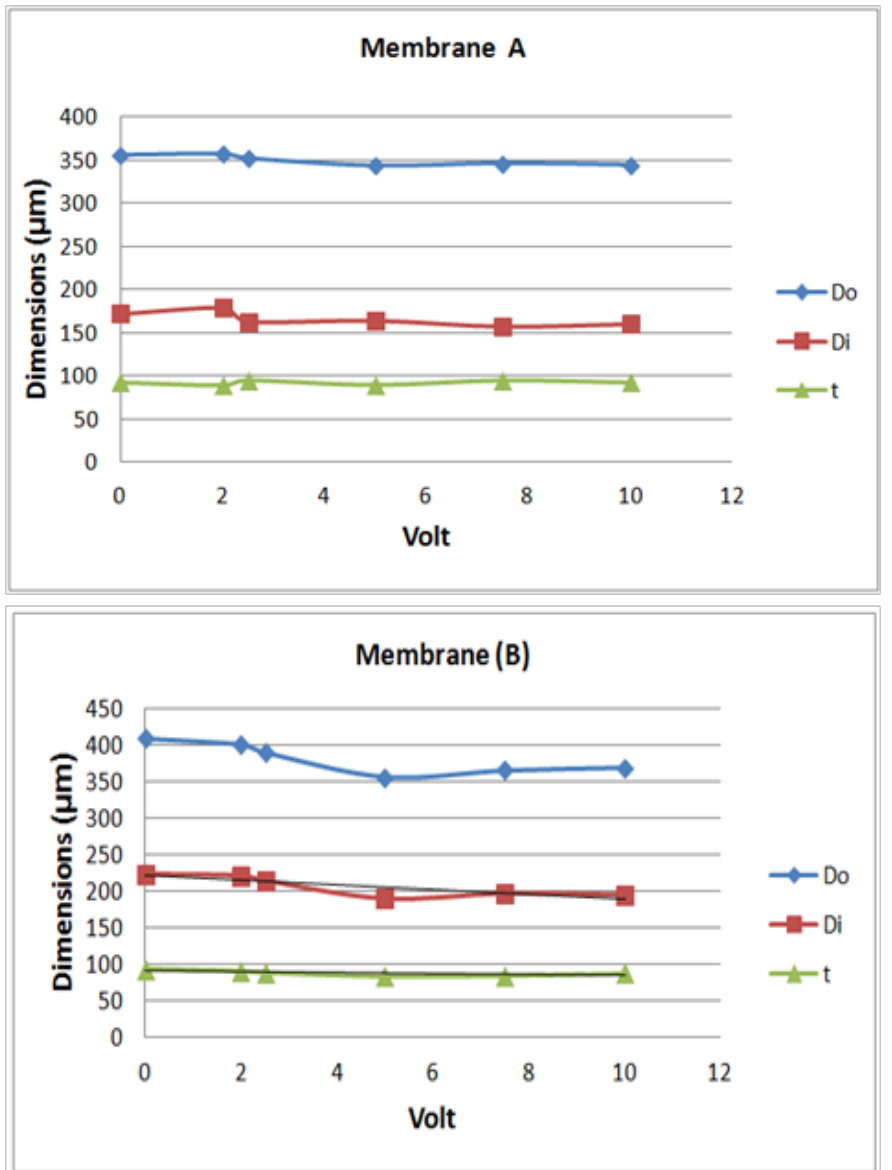

Figure 4 Effects of the voltage applied to membranes dimensions for membranes $A$ and $B$.

Table 3 shows the percentage change of dimensions of membranes (A) and (B) after treatment in alkaline medium at different applied voltages. Membrane contraction in all dimensions was observed in 5 -volt electrochemical treatment for both membrane types. Calculating method of the percentage change from the equation below:

$$
\frac{Y_{2}-Y_{1}}{Y_{1}} * 100
$$

Where, $Y_{1}$, Untreated membrane dimensions; $Y_{2}$, Membrane dimensions after electrochemical treatment

Table 3 Percentage change of dimensions in membranes A and B after treatment in alkaline medium.

\begin{tabular}{|c|c|c|c|}
\hline \multicolumn{4}{|c|}{ Percentage change of dimensions } \\
\hline Treatment voltage & $\Delta \mathrm{DO}(\%)$ & $\Delta$ DI (\%) & $\Delta \mathbf{t}(\%)$ \\
\hline \multicolumn{4}{|l|}{ Membrane raw A } \\
\hline 2 volts & 0 & 4 & -3 \\
\hline 2.5 volts & -1 & -5 & 3 \\
\hline 5 volts & -4 & -5 & -2 \\
\hline 7.5 volts & -3 & -9 & 3 \\
\hline 10 volts & -3 & -7 & 0 \\
\hline Membrane raw B & & & \\
\hline
\end{tabular}

Table Continued

\begin{tabular}{llll} 
Percentage change of dimensions & & \\
\hline Treatment voltage & $\Delta$ DO (\%) & $\Delta$ DI (\%) & $\Delta \mathbf{t}(\%)$ \\
2 volts & -2 & -1 & -3 \\
2.5 volts & -5 & -4 & -5 \\
5 volts & -13 & -15 & -11 \\
7.5 volts & -11 & -12 & -14 \\
10 volts & -10 & -13 & -6
\end{tabular}

Table 4 shows examples of the calculating method of percentage change after 5 and 7.5 volts treatment.

Table 4 Calculating method of percentage change.

\begin{tabular}{lllll}
\hline Treatment voltage & Dimensions & yl & y2 & $\begin{array}{l}\text { y2-y } \mathbf{l} \\
\mathbf{y} \mid * 100\end{array}$ \\
\hline Membrane (A) & & & & \\
5volts & DO & 356 & 343 & -4 \\
& DI & 172 & 164 & -5 \\
& t & 92 & 90 & -2 \\
7.5 volts & DO & 356 & 346 & -3 \\
& DI & 172 & 157 & -9 \\
Membrane (B) & t & 92 & 95 & 3 \\
5 volts & & & & \\
& DO & 409 & 356 & -13 \\
& DI & 223 & 190 & -15 \\
7.5 volts & t & 93 & 83 & -11 \\
& DO & 409 & 365 & -11 \\
& DI & 223 & 197 & -12 \\
& t & 93 & 80 & -14 \\
\hline
\end{tabular}

\section{Conclusion}

This work focused on the electrochemical treatment impacts on polysulfone (PS) HF membrane surface morphology, chemical composition and comparing these results with the untreated membrane surface. The results indicated the ability of the electric field to change the membrane surface morphology as membrane dimensions can be compacted or expanded as confirmed by SEM analysis. Maximum variations of dimensions DO, DI and thickness were $13 \%, 15 \%$, and $11 \%$ respectively obtained at 5 -volt treatment. Higher potential voltage above 2.5 volts can damage membrane surface and form cracks in it which were observed with the aid of SEM experiments. Electrochemical treatment can also change the membrane surface composition and convert it to a relatively selective membrane as it had imparted new sodium ions on the membrane surface as confirmed by EDX experiments. The best conditions were 2.5-volt electrochemical treatment and 1-hour time duration for which the highest indication of sodium mass percent was about $0.84 \%$ with no deformation detected. Porosity measurements showed no significant change between raw and treated membranes. These preliminary findings indicate the potential of electric fields to control membrane properties which suggest green strategies for membrane properties tuning. Current research is underway to explore changes in an acidic environment and also to identify flux and tensile changes for HF membranes with controlled additive content. 


\section{Acknowledgments}

None.

\section{Conflicts of interest}

The author declares that there are no conflicts of interest.

\section{References}

1. Chen JP. Membrane Separation: Basics and Applications. In: Membrane and Desalination Technologies, Wang LK, editors. Humana Press. Totowa, NJ. 2011;271-332.

2. Upadhyaya L, Qian X, Wickramasinghe SR. Chemical modification of membrane surface-an overview. Current opinion in chemical engineering. 2018;20:13-18.

3. Pinnau I, Freeman B. Formation and modification of polymeric membranes: an overview. Membrane Formation and Modification. 2000;744:1-22.

4. Akbari A, Homayonfal M, Jabbari V. Synthesis and characterization of composite polysulfone membranes for desalination in nanofiltration technique. Water Science and Technology. 2010;62(11):2655-2663.

5. Korminouri F. Surface modification of polysulfone hollow fiber membrane spun under different air-gap lengths for carbon dioxide absorption in the membrane contactor system. Chemical Engineering Journal. 2015;264:453-461.

6. Wenten I. Advances in polysulfone-based membranes for hemodialysis. Journal of Membrane Science and Research. 2016;2(2):78-89.

7. Kheirieh S, Asghari M, Afsari M. Application and modification of polysulfone membranes. Reviews in Chemical Engineering. 2017;34(5):10-23.

8. Abdelrasoul A. Morphology control of polysulfone membranes in filtration processes: a critical review. Chem Bio Eng Reviews. 2015;2(1):22-43.

9. Tafti AD. Optimized coupling of an intermittent DC electric field with a membrane bioreactor for enhanced effluent quality and hindered membrane fouling. Separation and Purification Technology. 2015;152:7-13.

10. Katuri KP. A novel anaerobic electrochemical membrane bioreactor (AnEMBR) with conductive hollow-fiber membrane for treatment of low-organic strength solutions. Environmental science \& technology. 2014;48(21):12833-12841.
11. Ensano BMB. Control of emerging contaminants by the combination of electrochemical processes and membrane bioreactors. Environmental Science and Pollution Research. 2019;26(2):1103-1112.

12. Ibeid S, Elektorowicz M, Oleszkiewicz JA. Novel electrokinetic approach reduces membrane fouling. Water research. 2013;47(16):6358-6366.

13. Li J, Ge Z, He Z. Advancing membrane bioelectrochemical reactor (MBER) with hollow-fiber membranes installed in the cathode compartment. Journal of Chemical Technology \& Biotechnology. 2014;89(9):1330-1336.

14. Chen JP. Study of the influence of the electric field on membrane flux of a new type of membrane bioreactor. Chemical Engineering Journal. 2007;128(2-3):177-180.

15. Coster H, Farahani TD, Chilcott T. Production and characterization of piezo-electric membranes. Desalination. 2011;283:52-57.

16. Darestani M, Coster H, Chilcott T. Piezoelectric membranes for separation processes: Operating conditions and filtration performance. Journal of membrane science. 2013;435:226-232.

17. Darestani M, Chilcott T, Coster H. Separation performance of PVDF membranes poled in intense electric fields. Separation and Purification Technology. 2013;118:604-611.

18. Darestani M, Chilcott $\mathrm{T}$, Coster $\mathrm{H}$. Changing the microstructure of membranes using an intense electric field: Filtration performance. Journal of membrane science. 2014;449:158-168.

19. McLaughlin S. The electrostatic properties of membranes. Annual review of biophysics and biophysical chemistry. 1989;18(1):113-136.

20. Vijh A, Conway B. Electrode kinetic aspects of the Kolbe reaction. Chemical Reviews. 1967;67(6):623-664.

21. Yuvaraj A, Santhanaraj D. A systematic study on electrolytic production of hydrogen gas by using graphite as an electrode. Materials Research. 2014;17(1):83-87.

22. NakhatePH, Joshi NT, Marathe KV.Acritical review of bioelectrochemical membrane reactor (BECMR) as cutting-edge sustainable wastewater treatment. Reviews in Chemical Engineering. 2017;33(2):143-161. 